\title{
Simplicial Perturbation Techniques and Effective Homology ${ }^{\star}$
}

\author{
Rocio Gonzalez-Díaz, Belén Medrano**, \\ Javier Sánchez-Peláez, and Pedro Real \\ Departamento de Matemática Aplicada I, \\ Universidad de Sevilla, Seville (Spain) \\ \{rogodi, belenmg, fjsp, real\}@us.es \\ http://www.us.es/gtocoma
}

\begin{abstract}
In this paper, we deal with the problem of the computation of the homology of a finite simplicial complex after an "elementary simplicial perturbation" process such as the inclusion or elimination of a maximal simplex or an edge contraction. To this aim we compute an algebraic topological model that is a special chain homotopy equivalence connecting the simplicial complex with its homology (working with a field as the ground ring).
\end{abstract}

\section{Introduction}

Simplicial complexes are widely used in geometric modelling. In order to classify them from a topological point of view, a first algebraic invariant that can be used is homology. We can cite two relevant algorithms for computing homology groups of a simplicial complex $K$ in $\mathbf{R}^{n}$ : The classical matrix algorithm 8 based on reducing certain matrices to their Smith normal form. And the incremental algorithm [1] consisting of assembling the complex, simplex by simplex, and at each step the Betti numbers of the current complex are updated. Both methods run in time at most $O\left(\mathrm{~m}^{3}\right)$, where $m$ is the number of simplices of the complex. Here, we deal with the problem of obtaining the homology $\mathcal{H}$ of a finite simplicial complex $K$ and a chain contraction (a special chain homotopy equivalence 8]) of the chain complex $C(K)$ to $\mathcal{H}$. This notion is a special case of effective homology [10. We call it an algebraic topological model for $K$ (or AT-model for $K)$.

Since the emergence of the Homological Perturbation Theory [5, 6], chain contractions have been widely used [10, 9, 5, 6]. The fundamental tool in this area is the Basic Perturbation Lemma (or BPL) which can be seen as a real algorithm such that the input is a chain contraction between two chain complexes $(C, d)$ and $\left(C^{\prime}, d^{\prime}\right)$ and a perturbation $\delta$ of $d$. The output is a chain contraction between the perturbed chain complexes $(C, d+\delta)$ and $\left(C^{\prime}, d^{\prime}+d_{\delta}\right)$. Here, we are interested

\footnotetext{
* Partially supported by the PAICYT research project FQM-296 "Computational Topology and Applied Math" from Junta de Andalucía.

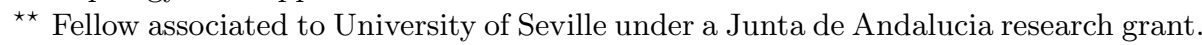


in the following complementary problem: given a chain contraction between two chain complexes $(C, d)$ and $\left(C^{\prime}, d^{\prime}\right)$ and a "perturbation" of $C$, is it possible to obtain a chain contraction between the perturbed chain complexes $(\tilde{C}, \tilde{d})$ and $\left(\tilde{C}^{\prime}, \tilde{d}^{\prime}\right)$ ?. Being the ground ring any field, Algorithms [1, 3 and 4 of this paper are positive answers to this question in the particular case of AT-models for simplicial complexes and "elementary simplicial perturbations" such as inclusion or elimination of a maximal simplex or an edge contraction. Moreover, Algorithm 1 is a version for AT-models of the incremental algorithm developed in [1]. It is described in [2, 3, 4] when the ground ring is $\mathbf{Z} / 2 \mathbf{Z}$ and applied to Digital Images for computing digital cohomology information.

\section{Algebraic Topological Models}

Now, we give a brief summary of concepts and notations. The terminology follows Munkres book [8]. For the sake of clarity and simplicity, we only will define the concepts that are really essential in this paper. We will consider that the ground ring is any field.

Simplicial Complexes. Considering an ordering on a vertex set $V$, a $q$-simplex with $q+1$ affinely independent vertices $v_{0}<\cdots<v_{q}$ of $V$ is the convex hull of these points, denoted by $\left\langle v_{0}, \ldots, v_{q}\right\rangle$. If $i<q$, an $i$-face of $\sigma$ is an $i$-simplex whose vertices are in the set $\left\{v_{0}, \ldots, v_{q}\right\}$. A simplex is maximal if it does not belong to any higher dimensional simplex. A simplicial complex $K$ is a collection of simplices such that every face of a simplex of $K$ is in $K$ and the intersection of any two simplices of $K$ is a face of each of them or empty. The set of all the $q$-simplices of $K$ is denoted by $K^{(q)}$. The dimension of $K$ is the dimension of the highest dimensional simplex in $K$.

Chains and Homology. Let $K$ be a simplicial complex. A $q$-chain $a$ is a formal sum of simplices of $K^{(q)}$. The $q$-chains form the qth chain group of $K$, denoted by $C_{q}(K)$. The boundary of a $q$-simplex $\sigma=\left\langle v_{0}, \ldots, v_{q}\right\rangle$ is the $(q-1)-$ chain: $\partial_{q}(\sigma)=\sum_{i=0}^{q}(-1)^{i}\left\langle v_{0}, \ldots, \hat{v}_{i}, \ldots, v_{q}\right\rangle$, where the hat means that $v_{i}$ is omitted. By linearity, $\partial_{q}$ can be extended to $q$-chains. The collection of boundary operators connect the chain groups $C_{q}(K)$ into the chain complex $C(K): \cdots \stackrel{\partial_{2}}{\rightarrow}$ $C_{1}(K) \stackrel{\partial_{1}}{\rightarrow} C_{0}(K) \stackrel{\partial_{0}}{\rightarrow} 0$. An essential property is that $\partial_{q} \partial_{q+1}=0$. In a more general framework, a chain complex $\mathcal{C}$ is a sequence $\cdots \stackrel{d_{2}}{\longrightarrow} C_{1} \stackrel{d_{1}}{\longrightarrow} C_{0} \stackrel{d_{0}}{\longrightarrow} 0$ of abelian groups $C_{q}$ and homomorphisms $d_{q}$, such that for all $q, d_{q} d_{q+1}=0$. The set of all the homomorphisms $d_{q}$ is called the differential of $\mathcal{C}$. A $q$-chain $a \in C_{q}$ is called a $q-$ cycle if $d_{q}(a)=0$. If $a=d_{q+1}\left(a^{\prime}\right)$ for some $a^{\prime} \in C_{q+1}$ then $a$ is called a $q$-boundary. Denote the groups of $q$-cycles and $q$-boundaries by $Z_{q}$ and $B_{q}$ respectively. We say that $a$ is a representative $q-$ cycle of a homology generator $\alpha$ if $\alpha=a+B_{q}$. Define the qth homology group to be the quotient group $Z_{q} / B_{q}$, denoted by $H_{q}(\mathcal{C})$. The $q$ th Betti number $\beta_{q}$ is the rank of $H_{q}(\mathcal{C})$. Intuitively, $\beta_{0}$ is the number of components of connected pieces, $\beta_{1}$ is the number of independent "holes" and $\beta_{2}$ is the number of "cavities". 
Chain Contractions [7]. A chain contraction of a chain complex $\mathcal{C}$ to another chain complex $\mathcal{C}^{\prime}$ is a set of three homomorphisms $(f, g, \phi)$ such that: $f: \mathcal{C} \rightarrow \mathcal{C}^{\prime}$ and $g: \mathcal{C}^{\prime} \rightarrow \mathcal{C}$ are chain maps; $f g$ is the identity map of $\mathcal{C}^{\prime} ; \phi: \mathcal{C} \rightarrow \mathcal{C}$ is a chain homotopy of the identity map $i d$ of $\mathcal{C}$ to $g f$, that is, $\phi \partial+\partial \phi=i d-g f$. Moreover, the annihilation properties $f \phi=0, \phi g=0$ and $\phi \phi=0$ are required. Important properties of chain contractions are: $\mathcal{C}^{\prime}$ has fewer or the same number of generators than $\mathcal{C} ; \mathcal{C}$ and $\mathcal{C}^{\prime}$ have isomorphic homology groups.

AT-model. Given a field as the ground ring, an AT-model for a simplicial complex $K$ is the set $(K, h, f, g, \phi)$ where $h$ is a set of generators of a chain complex $\mathcal{H}$ isomorphic to the homology of $K$ and $(f, g, \phi)$ is a chain contraction of $C(K)$ to $\mathcal{H}$. This implies that $f \partial=0$ and $\partial g=0$.

First Basic Perturbation Lemma (BPL) [5, 6]. Let $(f, g, \phi)$ be a chain contraction of the chain complex $(C, d)$ to the chain complex $\left(C^{\prime}, d^{\prime}\right)$. Let $\delta: C \rightarrow C$ be a morphism of degree -1 , called perturbation, such that $\phi \delta$ is pointwise nilpotent and $(d+\delta)^{2}=0$. Then $\left(f_{\delta}, g_{\delta}, \phi_{\delta}\right)$ given by $f_{\delta}=f-f \delta \Delta \phi, \phi_{\delta}=\Delta \phi$, $g_{\delta}=\Delta g$ is a chain contraction of $(C, d+\delta)$ to $\left(C^{\prime}, d^{\prime}+d_{\delta}\right)$ where $d_{\delta}^{\prime}=f \delta \Delta \phi$ and $\Delta=\sum_{i=0}^{\infty}(-1)^{i}(\phi \delta)^{i}$.

\section{Simplicial Perturbations on AT-Models}

Given a simplicial complex $K$ with $m$ simplices, an elementary simplicial perturbation of $K$ is one of these operations: an inclusion of a simplex, a deletion of a simplex, an edge contraction. Algorithms 103 and 4 compute AT-models after elementary simplicial perturbation. Moreover, the first one is a particular application of the BPL, showing in this way that there is a relation between the simplicial perturbation (where the ground graded groups are changed) and the algebraic perturbation (the original one, where just the differential changes).

Theorem 1. Given an AT-model for $K$ and an elementary simplicial perturbation of $K$, an AT-model for the perturbed complex $\tilde{K}$ can be computed using Algorithm 1, 可 or 4 in $\mathcal{O}\left(\mathrm{m}^{2}\right)$.

It is necessary to say that if we do not have an AT-model of $K$ as input, the computation of an AT-model of $\tilde{K}$ can be done applying Algorithm $1 m+1$ times in the worst case, so the complexity, in this case, is $\mathcal{O}\left(\mathrm{m}^{3}\right)$.

\subsection{AT-Models After Adding a Simplex}

An incremental algorithm for computing AT-models with coefficients in $\mathbf{Z} / \mathbf{2 Z}$ appears in 2, 3, 4. Here, we give an extension of the algorithm with coefficients in any field and prove that it is a particular application of BPL.

Given a chain $a$ and a simplex $\sigma$, define $c_{\sigma}(a)$ as the coefficient of $\sigma$ in $a$. 
Algorithm 1. Incremental algorithm for computing AT-models for simplicial complexes in any dimension with coefficients in any field.

InPUT: An AT-model $(K, h, f, g, \phi)$ for $K$ and a $q$-simplex $\sigma$ not in $K$ such that $K \cup\{\sigma\}$ is a simplicial complex.

If $f \partial(\sigma)=0$, then

$h:=h \cup\{\sigma\}, f(\sigma):=\sigma, g(\sigma):=\sigma-\phi \partial(\sigma)$ and $\phi(\sigma):=0$.

Else let $\beta \in h$ such that $\lambda:=c_{\beta}(f \partial(\sigma)) \neq 0$, then

$h:=h \backslash\{\beta\}, f(\sigma):=0, \phi(\sigma):=0$.

For every $\mu \in K$

If $\lambda_{\mu}:=c_{\beta}\left(f{ }_{K}(\mu)\right) \neq 0$ then

$f(\mu):=f(\mu)-\lambda_{\mu} \lambda^{-1} f \partial(\sigma)$,

$\phi(\mu):=\phi(\mu)+\lambda_{\mu} \lambda^{-1}(\sigma-\phi \partial(\sigma))$.

End if.

End for.

End if.

Output: The set $(K \cup\{\sigma\}, h, f, g, \phi)$.

Theorem 2. The output of Algorithm 1 defines an AT-model for $K \cup\{\sigma\}$.

Proof. Use BPL considering two cases.

- If $f \partial(\sigma)=0$, let $\mathcal{C}$ be the chain complex generated by the simplices of $K \cup\{\sigma\}$ with differential $d$ given by $d(\mu):=\partial(\mu)$ if $\mu \in K$ and $d(\sigma):=0$. Let $\mathcal{H}$ be the chain complex with null differential generated by $h \cup\{\sigma\}$. Let $\left(f^{\prime}, g^{\prime}, \phi^{\prime}\right)$ be the chain contraction of $\mathcal{C}$ to $\mathcal{H}$ defined by $f^{\prime}(\mu):=f(\mu)$ and $\phi^{\prime}(\mu):=\phi(\mu)$ if $\mu \in K ; g^{\prime}(\alpha):=g(\alpha)$ if $\alpha \in h ; f^{\prime}(\sigma):=\sigma, g^{\prime}(\sigma):=\sigma$ and $\phi^{\prime}(\sigma):=0$. Let $\delta: \mathcal{C} \rightarrow \mathcal{C}$ be defined by $\delta(\mu):=0$ if $\mu \in K$ and $\delta(\sigma):=\partial(\sigma)$. It is easy to see that $\phi^{\prime} \delta$ is pointwise nilpotent (since $\left.\delta \phi^{\prime} \delta=0\right)$ and $(d+\delta)^{2}=$ 0 . Apply BPL to the morphism $\delta$ and the chain contraction $\left(f^{\prime}, g^{\prime}, \phi^{\prime}\right)$ of $\mathcal{C}$ to $\mathcal{H}$ to obtain the chain contraction $\left(f_{\delta}, g_{\delta}, \phi_{\delta}\right)$ of $C(K \cup\{\sigma\})$ to $\mathcal{H}$.

Let us prove now that $\left(f_{\delta}, g_{\delta}, \phi_{\delta}\right)$ is the chain contraction obtained in Algorithm 1. We have that $f_{\delta}(\mu)=\left(f^{\prime}-f^{\prime} \delta \phi^{\prime}\right)(\mu)=f(\mu)$ and $\phi_{\delta}(\mu)=$ $\left(\phi^{\prime}-\phi^{\prime} \delta \phi^{\prime}\right)(\mu)=\phi(\mu)$ if $\mu \in K ; f_{\delta}(\sigma)=\left(f^{\prime}-f^{\prime} \delta \phi^{\prime}\right)(\sigma)=\sigma$ and $\phi_{\delta}(\sigma)=$ $\left(\phi^{\prime}-\phi^{\prime} \delta \phi^{\prime}\right)(\sigma)=0$; Finally, $g_{\delta}(\alpha)=\left(g^{\prime}-\phi^{\prime} \delta g^{\prime}\right)(\alpha)=g(\alpha)$ if $\alpha \in h$ and $g_{\delta}(\sigma)=\left(g^{\prime}-\phi^{\prime} \delta g^{\prime}\right)(\sigma)=\sigma-\phi \partial(\sigma)$.

- If $f \partial(\sigma) \neq 0$, let $\beta \in h$ such that $\lambda:=c_{\beta}(f \partial(\sigma)) \neq 0$. Let $\mathcal{C}$ be the chain complex generated by the set $K \cup\{\sigma, e\}$ where $e$ is an element of dimension $q-1$ that will be eliminated at the end. The differential $d$ of $\mathcal{C}$ is given by: $d(\mu):=\partial(\mu)$ if $\mu \in K, d(\sigma):=e$ and $d(e):=0$. Let $\mathcal{H}$ be the chain complex with null differential generated by $h$. Let $\left(f^{\prime}, g^{\prime}, \phi^{\prime}\right): \mathcal{C} \rightarrow \mathcal{H}$ be the chain contraction given by $f^{\prime}(e):=0, f^{\prime}(\sigma):=0, f^{\prime}(\mu):=f(\mu)$ if $\mu \in K ; g^{\prime}(\beta):=g(\beta)-\lambda^{-1} e, g^{\prime}(\alpha):=g(\alpha)$ if $\alpha \in h ; \phi^{\prime}(e):=\sigma, \phi^{\prime}(\sigma):=0$, $\phi^{\prime}(\mu):=\phi(\mu)+\lambda_{\mu} \lambda^{-1} \sigma$ if $\mu \in K$ and $\lambda_{\mu}:=c_{\beta}(f(\mu)) \neq 0$ and $\phi^{\prime}(\mu):=\phi(\mu)$ if $\mu \in K$ and $c_{\beta}(f(\mu))=0$. Let $\delta: \mathcal{C} \rightarrow \mathcal{C}$ be defined by $\delta(\mu):=0$ if $\mu \in K$, $\delta(e):=0$ and $\delta(\sigma):=\partial(\sigma)-e$. 
It is easy to see that $\phi^{\prime} \delta$ is pointwise nilpotent (since $\delta \phi^{\prime} \delta=0$ ) and $(d+\delta)^{2}=0$. Apply the BPL to the morphism $\delta$ and the chain contraction $\left(f^{\prime}, g^{\prime}, \phi^{\prime}\right)$ of $\mathcal{C}$ to $\mathcal{H}$ to obtain the chain contraction $\left(f_{\delta}, g_{\delta}, \phi_{\delta}\right)$ of $\mathcal{C}^{\prime}$ to $\mathcal{H}$ where $\mathcal{C}^{\prime}$ is generated by $K \cup\{\sigma\} \cup\{e\}$, with differential $d+\delta$.

Let us prove now that $\left(f_{\delta}, g_{\delta}, \phi_{\delta}\right)$ of $C(K \cup\{\sigma\})$ to $\mathcal{H}^{\prime}$ (generated by $h \backslash\{\beta\}$ with null differential) is the chain contraction obtained in Algorithm 1. We have that $f_{\delta}(\sigma)=\left(f^{\prime}-f^{\prime} \delta \phi^{\prime}\right)(\sigma)=0 ; f_{\delta}(\mu)=f(\mu)-\lambda_{\mu} \lambda^{-1} f \partial(\sigma)$ if $\mu \in K$ and $c_{\beta}(f \partial(\mu)) \neq 0$ and $f_{\delta}(\mu)=f(\mu)$ if $\mu \in K$ and $c_{\beta}(f \partial(\mu))=0$. On the other hand, $g_{\delta}(\alpha)=\left(g^{\prime}-\phi^{\prime} \delta g^{\prime}\right)(\alpha)=g(\alpha)$ if $\alpha \in h \backslash\{\beta\}$. Finally, $\phi_{\delta}(\sigma)=\left(\phi^{\prime}-\phi^{\prime} \delta \phi^{\prime}\right)(\sigma)=0 ; \phi_{\delta}(\mu)=\phi(\mu)+\lambda_{\mu} \lambda^{-1}(\sigma-\phi \partial(\sigma))$ if $\mu \in K$ and $\lambda_{\mu}=c_{\beta}(f \partial(\mu)) \neq 0$ and $\phi_{\delta}(\mu)=\phi(\mu)$ if $\mu \in K$ and $\lambda_{\mu}=c_{\beta}(f \partial(\mu))=0$.
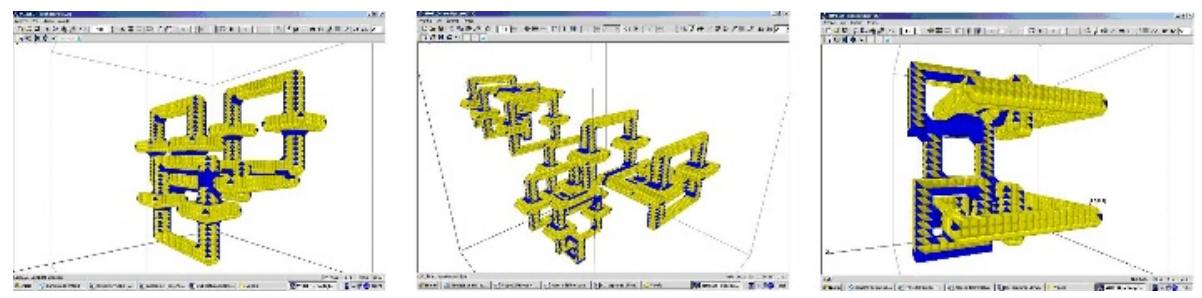

Fig. 1. The simplicial complexes $A, B$ and $C$

Using the algorithm above it is possible to design a procedure for computing AT-models for finite simplicial complexes in any dimension with coefficients in any field.
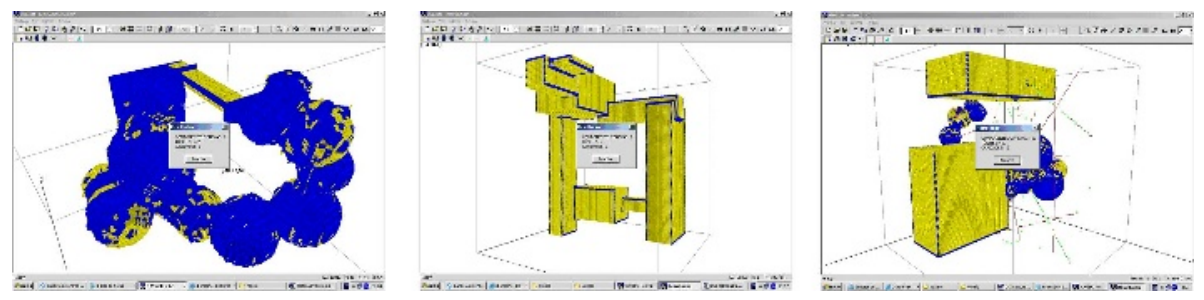

Fig. 2. The simplicial complexes $D, E$, and $F$

Algorithm 2. Computing an AT-model for a simplicial complex of any dimension with coefficients in any field.

INPUT: A simplicial complex $K$ and an ordered-by-increasing-dimension set of all the simplices of $K:\left\{\sigma_{0}, \ldots, \sigma_{n}\right\}$. 


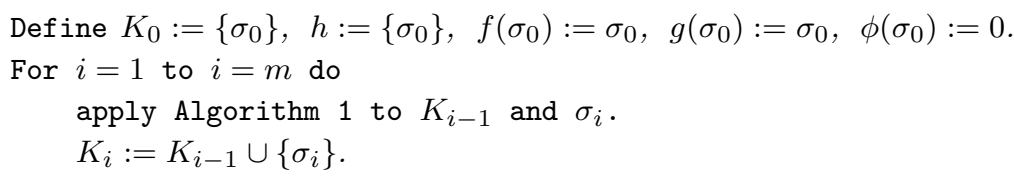

End for.

Output: An AT-model $\left(K_{m}, h, f, g, \phi\right)$ for $K$.

The implementation of the algorithm described before has been made by J. Sánchez-Peláez and P. Real. In Figures 1, 2, 3, 4, and 5, examples of computations (using this implementation) of AT-models for three-dimensional simplicial complexes are shown.

Example 1. Consider the simplicial complexes $A, B, C, D, E$ and $F$ shown in Figures 1 and 2. In Figures 3, 4, and 5, representative cycles of the homology generators of these complexes are shown. In the following table, we present the Betti numbers obtained and the running time for computing AT-models for these simplicial complexes in a Pentium 4, 3.2 GHz, 1Gb RAM.
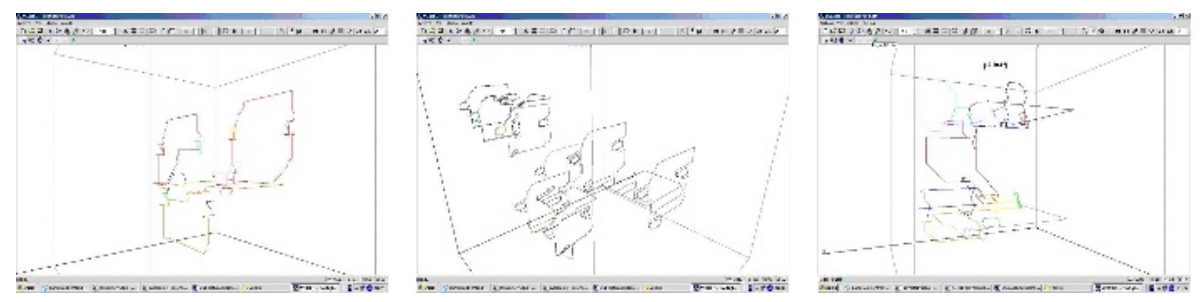

Fig. 3. The representative 1-cycles (holes) of $A, B$, and $C$

\begin{tabular}{|c|c|c|c|c|c|}
\hline Simplicial complex $K$ & Number of simplices of $K$ & Time & $\beta_{0}$ & $\beta_{1}$ & $\beta_{2}$ \\
\hline$A$ & 4586 & 7 seconds & 1 & 14 & 15 \\
$B$ & 13421 & 12 seconds & 3 & 46 & 39 \\
$C$ & 3286 & 4 seconds & 1 & 17 & 10 \\
$D$ & 18842 & 30 seconds & 1 & 27 & 5 \\
$E$ & 26308 & 50 seconds & 2 & 9 & 3 \\
$F$ & 31113 & 38 seconds & 138 & 419 & 13 \\
\hline
\end{tabular}

\subsection{AT-Models After Deleting a Maximal Simplex}

Now, an algorithm for computing an AT-model for a simplicial complex $K$ of any dimension with coefficients in any field after deleting a maximal simplex of $K$ is described. 

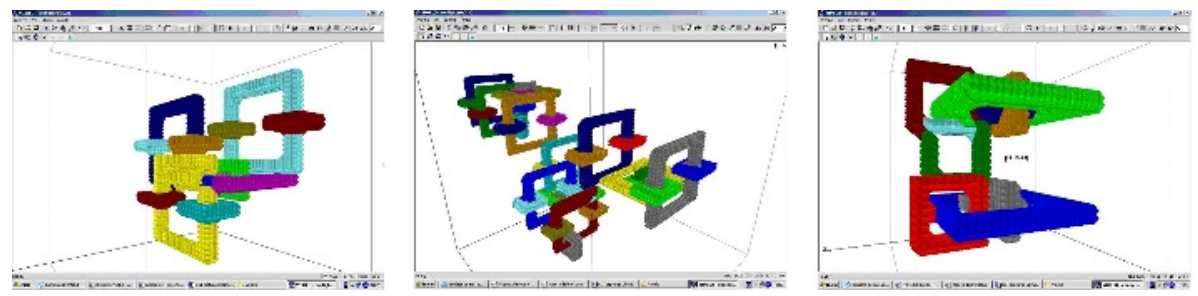

Fig. 4. The representative 2-cycles (cavities) of $A, B$, and $C$

Algorithm 3. Decremental algorithm for computing AT-models for simplicial complexes of any dimension with coefficients in any field.

INPUT: An AT-model $(K, h, f, g, \phi)$ for $K$ and a maximal $q$-simplex $\sigma$ in $K$.

If there exists $\beta \in h$ such that $\lambda:=c_{\sigma}(g(\beta)) \neq 0$, then

$h:=h \backslash\{\beta\}$.

For every $\mu \in K \backslash\{\sigma\}$ and $\alpha \in h$

If $\lambda_{\mu}:=c_{\beta}(f(\mu)) \neq 0$ then $f(\mu):=f(\mu)-\lambda_{\mu} \beta$.

End if.

If $\lambda_{\mu}^{\prime}:=c_{\sigma}(\phi(\mu)) \neq 0$ then $\phi(\mu):=\phi(\mu)-\lambda_{\mu}^{\prime} \lambda^{-1} g(\beta)$.

End if.

If $\lambda_{\alpha}:=c_{\sigma}(g(\alpha)) \neq 0$ then $g(\alpha):=g(\alpha)-\lambda_{\alpha} \lambda^{-1} g(\beta)$.

End if.

End for.

Else let $\gamma \in K$ be a $(q-1)$-simplex not in $h$ then

$h:=h \cup\{\gamma\}$ and $g(\gamma):=\partial(\sigma)$.

For every $\mu \in K \backslash\{\sigma\}$

If $\lambda_{\mu}^{\prime}:=c_{\sigma}(\phi(\mu)) \neq 0$ then

$f(\mu):=f(\mu)+\lambda_{\mu}^{\prime} \gamma$ and $\phi(\mu):=\phi(\mu)-\lambda_{\mu}^{\prime} \phi \partial(\sigma)$.

End if.

End for.

End if.

Output: The set $(K \backslash\{\sigma\}, h, f, g, \phi)$

Observe that the simplex $\gamma$ always exists since the morphism $f$ is onto and $f \partial(\sigma)=0$.

Theorem 3. The set $(K \backslash\{\sigma\}, h, f, g, \phi)$ defines an AT-model for $K \backslash\{\sigma\}$.

Proof. Let us denote by $\left(K, h_{K}, f_{K}, g_{K}, \phi_{K}\right)$ an AT-model for $K$ and by ( $K \backslash$ $\{\sigma\}, h, f, g, \phi)$ the AT-model for $K \backslash\{\sigma\}$ obtained using Algorithm 3. In order to prove that the output of Algorithm 3 is an AT-model for $K \backslash\{\sigma\}$, we check the two most important properties wich are $f g=i d$ and $i d-g f=\phi \partial+\partial \phi$. The rest of the properties are left to the reader. 

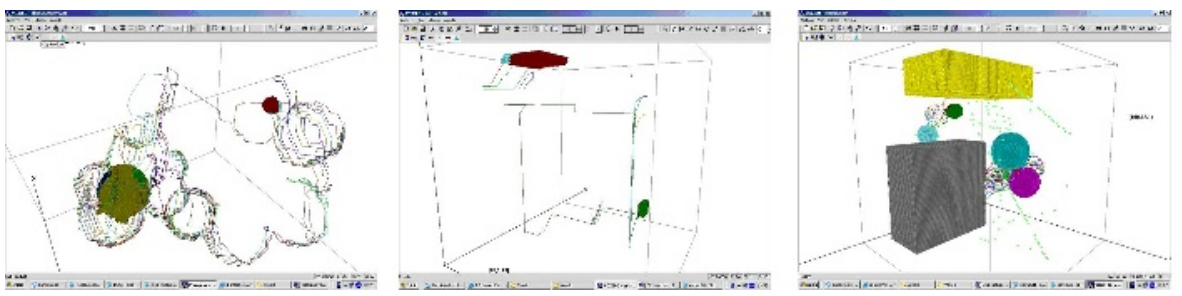

Fig. 5. The holes and cavities of $D, E$, and $F$

We distinguish two cases.

- If there exists $\beta \in h_{K}$ such that $\lambda:=c_{\sigma}(g(\beta)) \neq 0$ (recall that $\left.h:=h_{K} \backslash\{\beta\}\right)$.

- If $\alpha \in h$ such that $\lambda_{\alpha}:=c_{\sigma}\left(g_{K}(\alpha)\right) \neq 0$ then $f g(\alpha)=f\left(g_{K}(\alpha)-\right.$ $\left.\lambda_{\alpha} \lambda^{-1} g_{K}(\beta)\right)=f_{K} g_{K}(\alpha)-\lambda_{\alpha} \lambda^{-1}\left(f_{K} g_{K}(\beta)-\beta\right)=\alpha$.

Otherwise, $f g(\alpha)=f g_{K}(\alpha)=f_{K} g_{K}(\alpha)=\alpha$.

- If $\mu \in K \backslash\{\sigma\}$ and $\lambda_{\mu}^{\prime}:=c_{\sigma}\left(\phi_{K}(\mu)\right) \neq 0$ then $(\phi \partial+\partial \phi)(\mu)=\phi_{K} \partial(\mu)+$ $\partial\left(\phi_{K}(\mu)-\lambda_{\mu}^{\prime} \lambda_{1}^{-1} g_{K}\left(\alpha_{1}\right)\right)=\mu-g_{K} f_{K}(\mu)=\mu-g f_{K}(\mu)=\mu-g f(\mu)$.

If $c_{\sigma}\left(\phi_{K}(\mu)\right)=0$ and $\lambda_{\partial \mu}:=c_{\sigma}\left(\phi_{K} \partial(\mu)\right) \neq 0$ then $(\phi \partial+\partial \phi)(\mu)=$ $\phi_{K} \partial(\mu)-\lambda_{\partial \mu} \lambda_{1}^{-1} g_{K}\left(\alpha_{1}\right)+\partial \phi_{K}(\mu)=\mu-g_{K} f_{K}(\mu)-\lambda_{\partial \mu} \lambda_{1}^{-1} g_{K}\left(\alpha_{1}\right)=$ $\mu-g f(\mu)$.

In other case, $(\phi \partial+\partial \phi)(\mu)=\phi_{K} \partial(\mu)+\partial \phi_{K}(\mu)=\mu-g_{K} f_{K}(\mu)=$ $\mu-g f(\mu)$.

- Otherwise, let $\gamma \in K$ be a $(q-1)$-simplex not in $h_{K}$ (recall that $h:=h_{K} \cup\{\gamma\}$ and $g(\gamma):=\partial(\sigma))$.

- $f g(\gamma)=f \partial(\sigma)=f_{K} \partial(\sigma)+\gamma=\gamma$.

If $\alpha \in h_{K}, f g(\alpha)=f g_{K}(\alpha)=f_{K} g_{K}(\alpha)=\alpha$.

- If $\mu \in K \backslash\{\sigma\}$ and $\lambda_{\mu}^{\prime}:=c_{\sigma}\left(\phi_{K}(\mu)\right) \neq 0$ then $(\phi \partial+\partial \phi)(\mu)=\phi_{K} \partial(\mu)+$ $\partial \phi_{K}(\mu)-\lambda_{\mu} \partial \phi_{K} \partial(\sigma)=\mu-g_{K} f_{K}(\mu)-\lambda_{\mu} \partial(\sigma)=\mu-g f(\mu)$.

In other case, $(\phi \partial+\partial \phi)(\mu)=\phi_{K} \partial(\mu)+\partial \phi_{K}(\mu)=\mu-g_{K} f_{K}(\mu)=$ $\mu-g f(\mu)$.

Example 2. Let us show a simple example of the computation of an AT-model after deleting a maximal simplex. Consider a simplicial complex $K$ whose set of maximal simplices is $\{\langle 0,1,2\rangle,\langle 1,2,3\rangle\}$. The data of an AT-model for $K$ is showed in the following table.

\begin{tabular}{|c|c|c|c|c|}
\hline$K$ & $h$ & $f$ & $g$ & $\phi$ \\
\hline$\langle 0\rangle$ & $\langle 0\rangle$ & $\langle 0\rangle$ & $\langle 0\rangle$ & 0 \\
$\langle 1\rangle$ & & $\langle 0\rangle$ & & $\langle 0,1\rangle$ \\
$\langle 2\rangle$ & & $\langle 0\rangle$ & & $\langle 0,2\rangle$ \\
$\langle 3\rangle$ & & $\langle 0\rangle$ & & $\langle 0,1\rangle+\langle 1,3\rangle$ \\
$\langle 0,1\rangle$ & & 0 & & 0 \\
$\langle 0,2\rangle$ & & 0 & & 0 \\
$\langle 1,3\rangle$ & & 0 & 0 \\
$\langle 2,3\rangle$ & & 0 & & $\langle 1,2,3\rangle$ \\
$\langle 1,2\rangle$ & & 0 & & $\langle 0,1,2\rangle$ \\
$\langle 0,1,2\rangle$ & & 0 & & 0 \\
$\langle 1,2,3\rangle$ & & 0 & & 0 \\
\hline
\end{tabular}


After applying Algorithm 3 to the AT-model $(K, h, f, g, \phi)$ for $K$ and the maximal simplex $\langle 1,2,3\rangle$ we obtain the AT-model $(K \backslash\{\langle 1,2,3\rangle\}, h, f, g, \phi)$ for $K \backslash\{\langle 1,2,3\rangle\}$ whose data are:

\begin{tabular}{|c|c|c|c|c|}
\hline$K \backslash\{\langle 1,2,3\rangle\}$ & $h$ & $f$ & $g$ & $\phi$ \\
\hline$\langle 0\rangle$ & $\langle 0\rangle$ & $\langle 0\rangle$ & $\langle 0\rangle$ & 0 \\
$\langle 1\rangle$ & & $\langle 0\rangle$ & & $\langle 0,1\rangle$ \\
$\langle 2\rangle$ & & $\langle 0\rangle$ & & $\langle 0,2\rangle$ \\
$\langle 3\rangle$ & & $\langle 0\rangle$ & & $\langle 0,1\rangle+\langle 1,3\rangle$ \\
$\langle 0,1\rangle$ & & 0 & & 0 \\
$\langle 0,2\rangle$ & & 0 & & 0 \\
$\langle 1,3\rangle$ & & 0 & & 0 \\
$\langle 2,3\rangle$ & $\langle 2,3\rangle$ & $\langle 2,3\rangle$ & $\langle 2,3\rangle-\langle 1,3\rangle+\langle 1,2\rangle$ & $-\langle 0,1,2\rangle$ \\
$\langle 1,2\rangle$ & & 0 & & $\langle 0,1,2\rangle$ \\
$\langle 0,1,2\rangle$ & & 0 & & 0 \\
\hline
\end{tabular}

\subsection{AT-Models After Edge Contractions}

Finally, we deal with the problem of obtaining an AT-model for a simplicial complex $K$ after an edge contraction.

An edge contraction is given by the vertex map

$$
f^{(0)}: K^{(0)} \rightarrow L^{(0)}=K^{(0)}-\{\langle b\rangle\}
$$

where $f^{(0)}(\langle b\rangle)=\langle a\rangle$ and $f^{(0)}(\langle v\rangle)=\langle v\rangle$ for all $v \neq b$.

Let $K$ be a simplicial complex and $B$ a subset of $K$. Define

$$
\bar{B}=\left\{\sigma^{\prime} \in K: \sigma^{\prime} \text { is a face of } \sigma \in B\right\},
$$

St $B=\left\{\sigma \in K: \sigma^{\prime} \in B\right.$ is a face of $\left.\sigma\right\} \quad$ and $\quad L k B=\overline{S t B}-S t \bar{B}$.

The following algorithm computes an AT-model for a simplicial complex $K$ after and edge contraction in three steps. The goal of the first step is to obtain a chain contraction of $C(K)$ to the chain complex associated to a new simplicial complex (that we also denote by $K$ ) satisfying that $L k_{K}\{\langle a\rangle\} \cap L k_{K}\{\langle b\rangle\}=$ $L k_{K}\{\langle a, b\rangle\}$. In the second step, an AT-model $(K, h, f, g, \phi)$ for the new simplicial complex $K$ obtained at the first step is computed. At the final step, an AT-model for the simplicial complex $K$ after an edge contraction, by composing the chain contractions obtained at the previous steps is obtained.

Algorithm 4. Computing AT-models after edge contractions.

INPUT: An AT-model $A T:=\left(K, h_{K}, f_{K}, g_{K}, \phi_{K}\right)$ for $K$, an edge $\langle a, b\rangle \in K$ and an ordered-by-increasing-dimension set of all the simplices of $L k_{K}\{\langle a\rangle\} \cap L k_{K}\{\langle b\rangle\} \backslash L k_{K}\{\langle a, b\rangle\}$ : $\left\{\sigma_{1}=\left\langle w_{0}^{1}, \ldots, w_{m_{1}}^{1}\right\rangle, \ldots, \sigma_{n}=\left\langle w_{0}^{n}, \ldots, w_{m_{n}}^{n}\right\rangle\right\}$. 
STEP 1:

For $i=1$ to $i=n$ :

apply Algorithm 1 to the AT-model $A T$ and the simplex $\left\langle a, b, w_{0}^{i} \ldots, w_{m_{i}}^{i}\right\rangle$.

End for.

Define $K:=K \cup\left\{\left\langle a, b, w_{0}^{i}, \ldots, w_{m_{i}}^{i}\right\rangle\right\}$.

STEP 2:

For every simplex $\sigma \in K \backslash S t\{\langle b\rangle\}$ :

Define $f(\sigma):=\sigma, g(\sigma):=\sigma$ and $\phi(\sigma):=0$.

End for.

Define $f(\langle b\rangle):=\langle a\rangle, \quad \phi(\langle b\rangle):=\langle a, b\rangle, f(\langle a, b\rangle):=0$ and $\phi(\langle a, b\rangle):=0$.

For every simplex $\left\langle v_{0}, \ldots, v_{n}\right\rangle \in L k_{K}^{a}(b)$ :

$f\left(\left\langle b, v_{0}, \ldots, v_{n}\right\rangle\right):=\left\langle a, v_{0}, \ldots, v_{n}\right\rangle$ and $\phi\left(\left\langle b, v_{0}, \ldots, v_{n}\right\rangle\right):=0$.

If $n=0$ then $g\left(\left\langle a, v_{0}\right\rangle\right):=\left\langle b, v_{0}\right\rangle+\langle a, b\rangle$.

Else $g\left(\left\langle a, v_{0}, \ldots, v_{n}\right\rangle\right):=\left\langle b, v_{0}, \ldots, v_{n}\right\rangle+\sum_{S}(-1)^{i}\left\langle a, b, v_{0}, \ldots, \hat{v}_{i}, \ldots, v_{n}\right\rangle$

End if.

where $S=\left\{i: 0 \leq i \leq n,\left\langle v_{0}, \ldots, \hat{v}_{i}, \ldots, v_{n}\right\rangle \in L k_{K}\{\langle a, b\rangle\}\right\}$,

End for.

For every simplex $\left\langle v_{0}, \ldots, v_{n}\right\rangle \in L k_{K}\{\langle a, b\rangle\}$ :

$f\left(\left\langle a, b, v_{0}, \ldots, v_{n}\right\rangle\right):=0$ and $\phi\left(\left\langle a, b, v_{0}, \ldots, v_{n}\right\rangle\right):=0$,

$f\left(\left\langle b, v_{0}, \ldots, v_{n}\right\rangle\right):=\left\langle a, v_{0}, \ldots, v_{n}\right\rangle$ and $\phi\left(\left\langle b, v_{0}, \ldots, v_{n}\right\rangle\right):=\left\langle a, b, v_{0}, \ldots, v_{n}\right\rangle$,

End for.

$g\left(\left\langle a, v_{0}, \ldots, v_{n}\right\rangle\right):=\left\langle b, v_{0}, \ldots, v_{n}\right\rangle+\sum_{i=0}^{n}(-1)^{i}\left\langle a, b, v_{0}, \ldots, \hat{v}_{i}, \ldots, v_{n}\right\rangle$.

STEP 3:

Define $L:=\{f(\sigma): \sigma \in K\}$.

For every $\mu \in L$ and $\alpha \in h_{K}$ :

End for.

$f_{L}(\mu):=f_{K} g(\mu), \quad \phi_{L}(\mu):=f \phi_{K} g(\mu)$ and $g_{L}(\alpha):=f g_{K}(\alpha)$.

Output: The set $\left(L, h_{K}, f_{L}, g_{L}, \phi_{L}\right)$.

Here, we define by $L k_{K}^{a}(b)$ the set of all the simplices in $L k_{K}\{\langle b\rangle\}$ without having $a$ as a vertex. Moreover, without loss of generality, we suppose that $a<b<v$ for any vertex $v$ of $K$.

Theorem 4. Given a simplicial complex $K$ and an edge $\langle a, b\rangle \in K$, the set $\left(L, h_{K}, f_{L}, g_{L}, \phi_{L}\right)$ defines an AT-model for the simplicial complex $L$ obtained from $K$ after contracting the edge $\langle a, b\rangle$.

Proof. Let $\left(K, h_{K}, f_{K}, g_{K}, \phi_{K}\right)$ be an AT-model for a simplicial complex $K$ and let $\langle a, b\rangle$ be an edge in $K$. We only prove that the set $(K, h, f, g, \phi)$ (obtained at the second step) is an AT-model for the new simplicial complex $K$ (obtained at the first step). To this aim, we check that $f g=i d$ and $i d-g f=\phi \partial+\partial \phi$. The rest of the properties are left to the reader. 
- If $\sigma \in K \backslash S t\{\langle b\rangle\}$ then, by definition, $f g(\sigma)=\sigma$ and $(\phi \partial+\partial \phi)(\sigma)=0=$ $\sigma-g f(\sigma)$.

$-(\phi \partial+\partial \phi)(\langle b\rangle)=\partial(\langle a, b\rangle)=\langle b\rangle-\langle a\rangle=\langle b\rangle-g f(\langle b\rangle)$.

$(\phi \partial+\partial \phi)(\langle a, b\rangle)=\phi(\langle b\rangle-\langle a\rangle)=\langle a, b\rangle-g f(\langle a, b\rangle)$.

- If $\left\langle v_{0}, \ldots, v_{n}\right\rangle \in L k_{K}^{a}(b)$ then

- If $n=0, f g\left(\left\langle a, v_{0}\right\rangle\right)=f\left(\left\langle b, v_{0}\right\rangle+\langle a, b\rangle\right)=\left\langle a, v_{0}\right\rangle$.

$(\phi \partial+\partial \phi)\left(\left\langle b, v_{0}\right\rangle\right)=\phi\left(\left\langle v_{0}\right\rangle-\langle b\rangle\right)=-\langle a, b\rangle=\left\langle b, v_{0}\right\rangle-g f\left(\left\langle b, v_{0}\right\rangle\right)$.

- Otherwise,

$f g\left(\left\langle a, v_{0}, \ldots, v_{n}\right\rangle\right)=f\left(\left\langle b, v_{0}, \ldots, v_{n}\right\rangle+\sum_{S}(-1)^{i}\left\langle a, b, v_{0}, \ldots, \hat{v}_{i}, \ldots, v_{n}\right\rangle\right)$

$=\left\langle a, v_{0}, \ldots, v_{n}\right\rangle$.

$(\phi \partial+\partial \phi)\left(\left\langle b, v_{0}, \ldots, v_{n}\right\rangle\right)=\phi\left(\left\langle v_{0}, \ldots, v_{n}\right\rangle+\sum_{S}(-1)^{i+1}\left\langle b, v_{0}, \ldots, \hat{v}_{i}, \ldots, v_{n}\right\rangle\right)$

$=\sum_{S}(-1)^{i+1}\left\langle a, b, v_{0}, \ldots, \hat{v}_{i}, \ldots, v_{n}\right\rangle=(i d-g f)\left(\left\langle b, v_{0}, \ldots, v_{n}\right\rangle\right)$.

- If $\left\langle v_{0}, \ldots, v_{n}\right\rangle \in L k_{K}\{\langle a, b\rangle\}$ then

$f g\left(\left\langle a, v_{0}, \ldots, v_{n}\right\rangle\right)=f\left(\left\langle b, v_{0}, \ldots, v_{n}\right\rangle\right)=\left\langle a, v_{0}, \ldots, v_{n}\right\rangle$.

$(\phi \partial+\partial \phi)\left(\left\langle a, b, v_{0}, \ldots, v_{n}\right\rangle\right)=\phi\left(\left\langle b, v_{0}, \ldots, v_{n}\right\rangle\right)=\left\langle a, b, v_{0}, \ldots, v_{n}\right\rangle=(i d-$ gf $)\left(\left\langle a, b, v_{0}, \ldots, v_{n}\right\rangle\right)$.

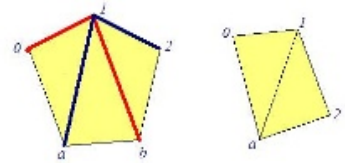

Fig. 6. Simplicial complexes $K$ and $L$

Example 3. Now we give a simple example of the computation of an AT-model after an edge contraction. Consider the simplicial complexes $K$ and $L$ (obtained from $K$ after contracting the edge $\langle a, b\rangle$ ) shown in Figure 6 . The simplices of $L k_{K}\{\langle a\rangle\}$ are in red, and the ones of $L k_{K}\{\langle b\rangle\}$ are in blue. In this case, $L k_{K}\{\langle a, b\rangle\}=L k_{K}\{\langle a\rangle\} \cap L k_{K}\{\langle b\rangle\}$. In the following table, the non-trivial results of the contraction $(f, g, \phi)$ from $C(K)$ to $C(L)$ (obtained using Algorithm4) are given:

\begin{tabular}{|c|c|c|c|c|}
\hline$K$ & $L$ & $f$ & $g$ & $\phi$ \\
\hline$\langle b\rangle$ & & $\langle a\rangle$ & & $\langle a, b\rangle$ \\
$\langle b, 1\rangle$ & & $\langle a, 1\rangle$ & & $\langle a, b, 1\rangle$ \\
$\langle b, 2\rangle$ & & $\langle a, 2\rangle$ & & 0 \\
$\langle a, b\rangle$ & & 0 & & 0 \\
$\langle a, b, 1\rangle$ & & 0 & & 0 \\
$\langle b, 1,2\rangle$ & & $\langle a, 1,2\rangle$ & & 0 \\
& $\langle a, 1\rangle$ & & $\langle b, 1\rangle+\langle a, b\rangle$ & \\
& $\langle a, 2\rangle$ & & $\langle b, 2\rangle+\langle a, b\rangle$ & \\
& $\langle a, 1,2\rangle$ & & $\langle b, 1,2\rangle+\langle a, b, 1\rangle$ & \\
\hline
\end{tabular}




\section{Conclusions}

Homological Perturbation Theory deals with algorithms for manipulating explicit chain homotopy equivalences between differential graded modules under suitable perturbations in the differentials of the modules. Here, we start a study for extending this theory to "module perturbations" produced in the underlying module structures. Working with a field as the ground ring, we reduce our perturbation homological analysis to particular chain contractions: AT-models for finite simplicial complexes. Taking as input an AT-model for a simplicial complex $K$ and perturbing it with changes in the graded module structure of $C(K)$ (addition or deletion of simplices or edge contractions), we have designed algorithms for restructuring the chain contraction information including these changes. More interesting simplicial perturbations such as "parallel" addition or elimination of simplices will be studied in a near future. Moreover, to extend these positive algorithmic results to general differential graded modules seems to be possible establishing an ordering for the generators of the underlying module structure.

\section{References}

1. Delfinado, C., Edelsbrunner, H.: An incremental algorithm for Betti numbers of simplicial complexes on the 3-sphere. Comput. Aided Geom. Design 12 (1995) $771-784$

2. González-Díaz, R., Real, P.: Towards digital cohomology. Lecture Notes in Computer Science 2886 (2003) 92-101

3. González-Díaz, R., Real, P.: On the cohomology of $3 D$ digital images. Discrete Applied Math 147 (2005) 245-263

4. Gonzalez-Diaz, R., Medrano, B., Real, P., Sánchez-Peláez, J.: Algebraic topological analysis of time-sequence of digital images. Lecture Notes in Computer Science 139 (2005) 208-219

5. Gugenheim, V.K.A.M. , Lambe, L., Stasheff, J.: Perturbation theory in differential homological algebra, II. Illinois J. Math. 35 (3) (1991) 357-373

6. Huebschmann, J., Kadeishvili, T.: Small models for chain algebras. Math. Z. 207 (1991) 245-280

7. MacLane, S.: Homology. Classic in Math., Springer-Verlag, Berlin, 1995

8. Munkres, J.R.: Elements of Algebraic Topology. Addison-Wesley Co. 1984

9. Real, P.: Homological perturbation theory and associativity. Homology, Homotopy and its Applications 2, No. 5 (2000) 51-88

10. Sergeraert, F.: The computability problem in algebraic topology. Adv. Math. 104, No. 1 (1994) 1-29 